\title{
Frank Greiner
}

\section{Les Amours romanesques de la fin des guerres de religion au temps de L’Astrée} (1585-1628)/ Fictions narratives et représentations culturelles

Paris : Honoré Champion, 2008, $560 \mathrm{p}$.

Comme le suggèrent son titre et son sous-titre, et ce, dès avant les principes énoncés très clairement dans l'introduction et rappelés en ouverture de la seconde partie (145), qui allient au repère littéraire un repère historique, ce livre ne se borne pas à prolonger les travaux des grands précurseurs (Reynier, Magendie ... ) sur son thème majeur, ni à tirer parti des nombreux ouvrages qui ont depuis quelques décennies mis en lumière un pan jusque-là peu exploré de notre production littéraire : le roman dit « baroque $»$. Frank Greiner a décidé de mettre en relation, ou plutôt de prendre en compte l'évidente relation qui existe entre fiction, sociologie, et histoire (événementielle, des mentalités, des civilisations, etc. ), et d’utiliser pour mener son enquête les outils propres à ces sciences aussi bien que ceux de l'analyse littéraire. Je constate avec satisfaction que les comportements ont évolué depuis 1978, où j’avais été sévèrement critiquée pour avoir donné comme sous-titre à ma thèse : « Analyse littéraire et histoire des mentalités » — mélange « impur », m’avait-on fait comprendre.

Aujourd'hui qu'il paraît au contraire nécessaire de ne pas considérer la littérature comme un monde clos, un chercheur (et un enseignant) en « lettres » — il faudra bien un jour revenir sur ces catégories! - peut donc produire pour obtenir son Habilitation à diriger des recherches un travail portant sur la culture, au sens large du terme, et d'une époque, en l'occurrence, sur cette culture que les « livres d'amour $\gg$ (l'expression était familière à Nervèze aussi bien qu'à Caseneuve, Du Bail et La Fontaine, alors que l'étiquette « roman sentimental » est apparue quelque deux siècles plus tard) nourrissent et véhiculent à la fois.

La première partie, dans un développement qui en compte cinq, commence ainsi par relever tout ce qu'ont en commun l'histoire de «l'éros baroque » et sa mise en fiction, puis s'occupe des hommes (auteurs et lecteurs, population de la Cour, des salons et divers cercles lettrés) ; la seconde s'arrête à la réception des œuvres (de la fin du XvI ${ }^{\mathrm{e}}$ siècle au romantisme), avant d'en étudier les textes et hors-textes.

La méthode employée dans la première partie appelle quelques réflexions. L'enquête historique (certes l'auteur ne se pose pas en historien, voir 27) demande une prudence à laquelle notre formation de littéraires ne nous a pas habitués : sélection des documents ; méfiance particulière à l'égard du « document » littéraire, mémoires, lettres et plus encore romans, qui ne sont utilisés qu’après avoir été vérifiés et soigneusement recoupés; respect du cadre chronologique défini, les regards sur 
ce qui s'est passé avant ou après n'étant destinés qu'à permettre des comparaisons ou des constats d'évolution, ou de rupture. Ici, les va et vient entre la réalité et les fictions, les déplacements dans le temps bien en-deçà ou au-delà des années 1585-1628 (de Marguerite de Navarre à Mme de Rambouillet, d'Érasme à Ninon de Lenclos ou au Sorel de la Connaissance des bons livres) laissent parfois songeur ; le survol est trop vite fait pour que les déductions auxquelles l'auteur aboutit ne paraissent pas sommaires. Mais pour réduire ce qui serait une faiblesse, Frank Greiner s'appuie, non sans les confronter avec un sens critique aiguisé, sur les travaux des meilleurs historiens d'hier et d'aujourd'hui : Jean Delumeau, Guy Bechtel, Philippe Ariès, François Bluche, etc. Il donne ainsi à son propre discours des garants suffisants pour qu'il inspire confiance - peut-être plus à son lecteur qu'à lui-même, qui dans un paragraphe conclusif (142), argumente encore (« après tout $\gg, \ll$ en définitive » ... ) sur la valeur des résultats de son enquête.

La suite n'appelle pas de réserves, le sujet y étant dominé par un auteur qui déclare d'ailleurs qu'au fil de son travail son impression de « diversité, liée à celle d'arbitraire », est allée en s'atténuant (148), et qui évolue à l'aise dans la compagnie des 113 écrivains de son corpus, ce qui représente une masse considérable de romans ou d'histoires' — la pièce majeure étant évidemment L'Astrée; mais la méthode adoptée justifie qu'aucun chapitre particulier ne lui soit réservé —, sans parler des autres lectures qui s'imposaient à lui, de Platon à Thomas d'Aquin, de Vivès à Montaigne et à Richelieu. En effet, caractériser une culture n'est pas une mince affaire : il faut récolter d'abondantes informations, et pour en rendre compte ne pas craindre de décrire en termes clairs.

C'est ce qui est fait ici, tout au long du développement et de manière systématique, à propos du roman : des scénarios et du décor aux formes et styles de l'imaginaire qui y est à l'œuvre aux valeurs qu'il véhicule et aux types. Est ainsi mis en évidence le « jeu d'affinités littéraires » que notre collègue désigne comme son « fil d'Ariane » (148). Ainsi retrouve-t-on, comme on s'y attendait, les histoires «tragiques », « exemplaires » ou (et) « dévotes » (la thèse de Jean Descrains aurait dû servir à l'étude de Jean-Pierre Camus), les « aventures d'armes et d'amour » et le « roman grec $»$, et aussi ce que Frank Greiner rassemble sous le titre d'« amours courtoises $\gg$ qui a, malgré son allure anachronique, l'avantage de souligner une filiation que les découpages de l'histoire littéraire tendent par trop à camoufler.

Le développement sur les « valeurs », notion plus appropriée à l'époque que le concept moderne d'idéologie, aurait mérité de prendre de l'ampleur, fût-ce au détriment de l'équilibre arithmétique des chapitres. La problématisation du rapport entre les mœurs et les mentalités-fiction y apparaît très bien : dominantes 
et lieux communs se nuancent, notamment en fonction de l'attente des lecteurs et du mode de représentation adopté par l'écrivain; dans « l'espace virtuel » du roman, la norme, si présente qu'elle soit, est sujette, plus ou moins discrètement, à des incertitudes et remises en question, jusqu'à des « dissidences (373) » que la société ne connaît pas. Il y a là un filon à creuser, ce qui éviterait probablement un travers que le critique pointe du doigt à propos de L'Astrée, tout en le laissant à l'état d'intuition : elle ne fascinerait ses lecteurs modernes « qu'au prix d'un certain nombre de malentendus $\gg$ (149).

En quelques pages, Frank Greiner tire les conclusions de son enquête : lieux d'expression, selon les cas, de controverses (Nervèze, Rosset) ou de dialogue et de conciliations entre règle et passions (pulsions ?) individuelles (Urfé), les « livres d'amour $\gg$ lui apparaissent justement, dans leur instabilité caractéristique de la production baroque, comme « le dépôt d'un modèle en devenir » (493). Sans doute le lecteur reste-t-il un peu sur sa faim, mais l'essentiel est dit, et les pistes ouvertes lui permettront, ou permettront à d'autres d'approfondir et de prolonger l'étude.

C'est dire que cet ouvrage bien écrit (à quelques coquilles, négligences et bizarreries près), qui fournit une liste des auteurs de fictions sentimentales et une bibliographie abondante, et dont la lecture est facilitée par la présence de deux index (des noms et des titres), constitue un apport des plus utiles, à travers la connaissance d'un genre, celle de ce qu'on peut continuer à appeler, par commodité, l'époque baroque.

MADELEINe BeRTAUd, Université de Nancy

\section{Hélisenne de Crenne}

\section{Les Epitres familières et invectives, Le Songe}

Édition Jean-Philippe Beaulieu.

Collection La cité des dames, St-Étienne : Publications de l'Université de SaintEtienne, 2008, 188p.

Le format de ce volume impose certaines limites quant à la longueur de l'introduction, le nombre de notes, et l'exclusion d'un index des noms et des variantes, mais il n'est pas ici question de lacunes, bien au contraire. Euvre de travail ou non, cette édition, si concise soit-elle, est riche de notes en bas de page, présentant des références historiques ou littéraires, des équivalents simples aux passages souvent impénétrables pour la compréhension du style — disons alambiqué sinon amphigourique - d'Hélisenne de Crenne, mais aussi de nombreux éclaircissements, 